\title{
The spontaneous reporting of adverse drug reactions
}

\section{by nurses}

M. HALL, P. MCCORMACK, N. ARTHURS ${ }^{1}$ \& J. FEELY

Department of Pharmacology and Therapeutics, and Pharmacoepidemiology and Medicines Evaluation Unit, Trinity Centre for Health Sciences, St James's Hospital, Dublin 8 and ${ }^{1}$ National Drugs Advisory Board, 63 Adelaide Road, Dublin 2, Ireland

In an attempt to improve the low reporting rate of adverse drug reactions (ADR) we examined the potential for hospital nurses to report ADRs through a spontaneous 'yellow card' system. Over 14 months 100 cards were received (compared with 28 cards from doctors). Although reports from doctors for the same period were of a more substantial nature, nurses nevertheless reported many life threatening $(17 \%)$ or moderately severe $(76 \%)$ reactions. Nurses identified uncertainty concerning their role and deficient in-service education on drug therapy as major constraints in their participation. Given their unique position in drug administration and recording observations on patients, we believe that nurses could contribute significantly and in a complementary fashion to the spontaneous reporting of adverse reactions.

Keywords adverse drug reactions spontaneous reporting

\section{Introduction}

Spontaneous (yellow card) reporting of ADRs remains the most widely used and cost effective surveillance system and is the cornerstone of safety monitoring of drugs in clinical practice. It may detect previously unrecognised adverse reactions and identify risk factors that pre-dispose to drug toxicity and investigate causality. In addition to identifying drug safety problems it may facilitate risk-benefit judgments and comparisons within therapeutic categories [1-3]. The free post yellow card system is in use in Ireland since 1968 and is similar to that in use in the United Kingdom (UK) and complies with World Health Organisation (WHO) guidelines. Approximately 1,800 cards are received annually from a population of 3.5 million.

There is however considerable international variation in the use of such systems with active participation from prescribers in the UK, Ireland and the Netherlands and considerably less in Germany and Belgium [4]. In the UK, there is also considerable regional variation and a number of initiatives, such as local coordinators, reporting centres and easier availability of cards through insertion in the British National Formulary, have enhanced the rate of reporting [1]. In Ireland our study [5] found that a reporting fee increased the number of ADR reports and also identified a role for nurses in such reporting. This study investigates the contribution of and constraints for nurses reporting ADRs.

\section{Methods}

This study was undertaken in Ireland in a 760 bed general teaching hospital with approximately 22,000 admissions (approximately 56\% female) annually. The background to the study was explained to nursing administration and senior nursing staff. An information package outlining the aims and objectives of the study, a definition of what an ADR is and guidelines on how to report, with the Adverse Reaction Report of the Irish regulatory agency, National Drugs Advisory Board (NDAB), was supplied to each ward. A research nurse held briefing meetings with Ward Sisters throughout the hospital and answered any queries. Nurses reported reactions using a 'yellow card' similar to the UK model, but were encouraged to report on all ADRs, not just selected ones. These wards were visited every 2 weeks and the spontaneous reports were collected.

Adverse reactions were defined as 'any response to a drug that is noxious and/or unintended and that occurs at dosage used in the main for prophylaxis, diagnosis or therapy, excluding the failure to accomplish the intended purpose'. The severity of the reaction was classified as outlined in Table 1.

Two forms of audit were employed; one internal and one external. An independent random audit of $30 \%$ of all ADRs was undertaken by a physician who was not given details of the report but was asked to review the patient. The external assessment of the quality of 
Table 1 Adverse drug reactions reported by nurses; definition of severity with examples

\begin{tabular}{|c|c|c|}
\hline Mild 7\% & Moderate $76 \%$ & Severe $17 \%$ \\
\hline $\begin{array}{l}\text { Incidental, required } \\
\text { no treatment and did } \\
\text { not necessarily call } \\
\text { for withdrawal of any } \\
\text { treatment }\end{array}$ & $\begin{array}{l}\text { Required admission to } \\
\text { hospital or prolonged } \\
\text { the stay in hospital by } \\
\text { at least } 1 \text { day }\end{array}$ & $\begin{array}{l}\text { Fatal or life } \\
\text { threatening. }\end{array}$ \\
\hline Examples: & Examples: & Examples: \\
\hline Inflammation at site- & Florid skin rash- & Anaphylactic reaction- \\
\hline Prochlorperazine & Trimethoprim & $\begin{array}{l}\text { Co-amoxiclav, } \\
\text { floxacillin, cefuroxime }\end{array}$ \\
\hline Nausea- & Vomiting- & Thrombocytopaenia- \\
\hline Amoxicillin & Erythromycin & $\begin{array}{l}\text { Co-trimoxazole, } \\
\text { heparin }\end{array}$ \\
\hline Rash- & Dystonic reaction- & Stevens Johnson syndrome- \\
\hline Co-amoxiclav & Metoclopramide & $\begin{array}{l}\text { Co-amoxiclav, } \\
\text { carbamazepine }\end{array}$ \\
\hline
\end{tabular}

reports involved a computer generated grading programme using established criteria [6] at the WHO Collaborating Centre (Uppsala).

\section{Results}

In the subsequent 14 months (November 1991 to January 1993), 100 adverse reactions were reported by nurses (examples Table 1) and 28 by doctors who usually report 20-30 per annum. The demography of patients and route of drug administration and principal system involved is shown in Table 2. Following a review of the patient and case notes, the independent audit physician found no evidence of reporting trivial ADR or a tenuous relationship and there was concordance in describing the association and severity in $90 \%$ of cases. There was only one case of duplication of reports between nurses and doctors, an anaphylactic reaction resulting in death. There were however interesting differences. The reports from nurses largely concerned antimicrobials (52\% total), drugs commonly given by non-oral routes $(52 \%)$ and dermatological reactions $(44 \%)$. Physicians implicated less antimicrobials $(11 \%)$ but more cardiovascular agents $(36 \%)$, more often drugs given orally $(85 \%)$ but fewer (15\%) dermatological effects and more investigational based diagnoses (interstitial pneumonitis-amiodarone, renal failure-diclofenac, hepatitis-metoclopramide). The WHO Collaborating Centre's assessment found nurses omitted significantly more $(P<$ 0.05 Chi Square test) important case information item(s) (treatment dates, reaction dates, identification of case, etc.) [6] than doctors $(61 \%$ vs $39 \%$ unassessable from our hospital doctors and a random sample of 60 doctors' reports outwith our hospital). Similarly fewer reports from nurses could be classified [6] as feasible or substantial reports $(4 \%$ and $31 \%$ vs $6 \%$ and $55 \%$ respectively). Nonetheless in numerical terms nurses reported more ( 31 vs $15, P<0.05$ ) substantial reports than doctors.

Following this study an attitudinal questionnaire was completed by nurses. Strong evidence of causation (new sign or symptom with disappearance on discontinuation) was felt necessary prior to reporting. Many nurses felt it was their duty to report such reactions to a doctor who would subsequently determine causality. Despite the additional constraint on their time and extra amount of paper work, most nurses (85\%) welcomed the opportunity to report reactions. There were consistent requests by nurses for additional information on drug therapy.

\section{Discussion}

In this study nurses reported 100 reports of ADR in 14 months. This compares with 28 medical reports from doctors at the same hospital. The majority of nurses' reports were either life threatening (17\%) or moderately serious $(76 \%)$. Understandably for a group new to the system the quality of reporting was judged by the WHO Collaborating Centre to be less than that of doctors in Ireland. Nevertheless the reports represented ADRs that had occurred, as shown by an independent medical audit, and overall the numbers of substantial reports was more than twice those reported by doctors. Furthermore, nurses reported a different variety of ADR than physicians, more associated with intravenous therapy, involving antibiotics and those producing skin reactions. The attitudinal questionnaire confirmed our impression that nurses are more likely to report reactions with obvious signs and require firm evidence of causality before making a report. On the other hand reports from doctors at our hospital related more to oral and chronic therapy, cardio-vascular drugs and include those requiring laboratory diagnosis-hepatitis, pneumonitis, nephropathy but fewer dermatological reactions. There was a similar proportion of serious/life threatening reactions. The complementary role of the professions is shown by the almost negligible duplicate reporting (one anaphylaxis) which also suggests considerable under-reporting by both groups. 
Table 2 Characteristics of patients experiencing adverse reactions, the route of drug administration and principle system involved

\begin{tabular}{lr}
\hline & $\%$ \\
\hline Patients & 38 \\
Male & 62 \\
Female & 78 \\
Medical & 22 \\
Surgical & \\
& \\
Route of administration & 48 \\
Oral & 42 \\
Intravenous & 5 \\
Topical & 3 \\
Intramuscular & 2 \\
Inhaled & \\
& \\
Manifestation of ADR & 44 \\
Skin & 24 \\
Gastrointestinal & 12 \\
Central nervous system & 6 \\
Cardiovascular & 5 \\
Haematology & 4 \\
Muscle/Joint & 4 \\
Anaphylaxis/Allergy & 1 \\
Respiratory & \\
\hline
\end{tabular}

There are good logistical reasons for involving nurses in the reporting system. Nurses administer most drugs, are commonly present when an adverse reaction occurs, have a well developed mechanism for recording their observations and generally are the source for alerting the prescriber. While we encountered initial hesitancy amongst nurses to participate, this largely reflected uncertainty regarding their role and lack of information on the adverse effects of drug therapy. This may contribute to a lesser quality report, a constraint that education and experience can help overcome. Similar uncertainty and unfamiliarity in the medical profession has been noted [5] which seems to

\section{References}

1 Rawlins MD. Spontaneous reporting of adverse drug reactions. Br J Clin Pharmacol 1988; 26: 1-5.

2 Dukes MNG. The importance of adverse reactions in drug regulation. Drug Safety 1990; 5: 3-6.

3 Rawlins MD. Pharmacovigilance: Paradise lost, regained or postponed. J Roy Coll Phys Lond 1995; 29: 41-49.

4 Wilholm B-E, Olsson S. Spontaneous reporting systems outside the United States. In Pharmacoepidemiology, ed Strom BL. New York: Churchill Livingstone, 1989: 119-134.

5 Feely J, Moriarty S, O'Connor P. Stimulating reporting of adverse drug reactions by using a fee. Br Med J 1990; be greater in hospital based doctors than in those in general practice [3]. These were greater constraints than any additional paper work involved and $85 \%$ of nurses indicated a wish to continue to participate in ADR reporting. The study also highlighted consistent requests by nursing staff for further information about drug therapy, identifying a major need in their continuing professional education.

With the establishment of a European Medicines Evaluation Agency, there is an increased focus on a common European initiative in pharmacovigilance and ADR reporting. Currently there is considerable international difference in reporting rates. Furthermore, the 'right to report' varies considerably from a completely open system, including patients in the United States, to a system restricted to doctors, dentists and coroners as in the United Kingdom. In Ireland where reports from pharmacists and nurses are accepted such restrictions do not exist but in practice few nurses report ADRs. Currently the potential role of pharmacists to report to the Committee on Safety of Medicines (CSM) is under study. Of interest, the European Medicines Evaluation Agency draft guidelines [7] envisage a role for pharmacists and nurses in reporting ADRs.

At present, less than $10 \%$ of all reactions are reported $[1,2]$. In our view opening access to reporting by nurses would enhance the process immediately quantitatively and in time qualitatively. Raising the report rate in a hospital setting where more new drugs and potentially toxic combinations are used should provide additional 'early alert' information. We believe that nurses with appropriate instruction in ADR reporting could add significantly to drug safety.

This study was supported by the Health Research Board. We wish to thank the nursing staff of St James's Hospital, the NDAB and WHO (Collaborating Centre, Uppsala) for their co-operation.

300: 22-23.

6 Edwards IR, Lindquist M, Wilholm B-E, Napke E. Quality criteria for early signals of possible adverse reactions. Lancet 1990; 336: 156-158.

7 European Communities Commission. Draft notice to applicants for marketing authorisations for medicinal products for human use in the European Union. Brussels: 1994 Commission Document III/5944.

(Received 19 September 1994, accepted 19 April 1994) 